\title{
Cosmic ray modulation as a possible diagnostic for the low-wavenumber behaviour of turbulence in the heliospheric magnetic field
}

\author{
N.E. Engelbrecht* \\ South African National Space Agency (SANSA) \\ E-mail: n.eugene.engelbrechtegmail.com
}

\section{R.A. Burger}

North-West University (Potchefstroom campus)

E-mail: Adri.Burgerenwu.ac.za

\begin{abstract}
Galactic cosmic-ray (CR) intensities calculated using numerical modulation models that proceed from first principles, where the diffusion tensor is calculated using as inputs observationally motivated forms for the heliospheric turbulence power spectrum as function of turbulence quantities yielded by turbulence transport models, are incredibly sensitive to assumptions made as to the low-wavenumber behaviour of the 2D turbulence power spectrum. In this study, a novel ab initio $\mathrm{CR}$ modulation code that solves a set of stochastic transport equations equivalent to the Parker transport equation is introduced, and employed to study this sensitivity. Preliminary results, including those pertaining to possible constraints on the low-wavenumber behaviour of the $2 \mathrm{D}$ power spectrum gleaned from computed CR spectra, will also be presented.
\end{abstract}

The 34th International Cosmic Ray Conference,

30 July- 6 August, 2015

The Hague, The Netherlands

\footnotetext{
* Speaker.
} 